\title{
Phase transition in dependent percolation
}

\author{
Christopher Hoffman ${ }^{1,2}$
}

\begin{abstract}
In this paper we discuss two different models of dependent percolation on the graph $\mathbb{Z}^{2}$. We show that they both exhibit phase transitions. This proves a conjecture of Jonasson, Mossel and Peres [6], who proved a similar result on $\mathbb{Z}^{3}$.
\end{abstract}

\section{Introduction}

Since mathematical models only approximate physical systems the stability of the model is important. A small change in the model should ideally result in a small change in the qualitative behavior of the model. In this paper we consider the stability of phase transitions in percolation. In Bernoulli percolation the existence of a phase transition is both one of the most important results and one of the most elementary. However, in the models of dependent percolation that have been studied, establishing a phase transition has been often quite difficult. In this paper we show that two models of dependent percolation exhibit phase transitions.

The pioneering work in studying the stability of statistical mechanics models was done by McCoy and $\mathrm{Wu}$ [9] [10]. They studied the Ising model in a random environment. Their models had interactions which depend only on a row or column. This produced models which were both tractable and had physically realistic results. In addition to the Ising model, people have studied other particle systems, such as the contact process and the voter model, in random environments [11] [7] [8] [3]. The models that we study in this paper are natural examples for percolation of this family of statistical mechanics models in a random environment.

Let us also note that in addition to the stability of statistical mechanics models, phase transitions in dependent percolation are also related to problems in distributive computing. In 1990 Winkler made a conjecture about colliding random walks on graphs involving 'clairvoyant demons'. Noga Alon reformulated this conjecture in terms of the existence of a phase transition in a simple model of oriented percolation. Before this conjecture was resolved progress was made on a number of related topics. Winkler [12] and Balister, Bollobas, and

\footnotetext{
${ }^{1}$ Department of Mathematics, University of Washington, Seattle, WA 98195 hoffman@math.washington.edu

${ }^{2}$ Research supported in part by an NSF postdoctoral fellowship
} 
Stacey [1] independently proved that there exists a phase transition for unoriented percolation in this model. Also Gacs answered a related question of compatibility of independent sequences [5]. Gacs recently proved Winkler's conjecture.[4]. The location of phase transition is still unknown.

The first of our models was introduced by Jonasson, Mossel and Peres [6]. This model is on the graph with vertices $\mathbb{Z}^{2}$ and with edges between nearest neighbors (i.e., there is an edge between $(x, y)$ and $\left(x^{\prime}, y^{\prime}\right)$ iff $\left|x-x^{\prime}\right|+\left|y-y^{\prime}\right|=1$.) To define the probability that an edge is included in the graph (or open) we define two sequences of random variables. Let

$$
\left\{x_{i}\right\}_{i \in \mathbb{Z}} \quad \text { and } \quad\left\{y_{i}\right\}_{i \in \mathbb{Z}}
$$

be i.i.d. random variables. They have geometric distribution with parameter $2^{-1000}$. Thus

$$
P\left(x_{i}>\lambda\right)=C\left(2^{-1000}\right)^{\lambda} .
$$

Let $p$ be a number $0<p<1$. Then set

$$
P_{p}(e((i, j),(i+1, j)) \text { is open })=p^{x_{i}}
$$

and

$$
P_{p}(e((i, j),(i, j+1)) \text { is open })=p^{y_{i}} .
$$

Each edge is either open or closed independently of all other edges conditioned on the values given above. We refer to this model as percolation on the randomly stretched lattice in $\mathbb{Z}^{2}$.

It is given this name for the following reason. Consider a graph generated by the coordinate axis and lines parallel to the coordinate axis as follows. The distance between the $i^{\text {th }}$ line to the right of the $y$ axis and the $i+1$ st line is $x_{i}$. The distance between the $j^{\text {th }}$ line to the left of the $y$ axis and the $j-1$ st line is $x_{-j}$. In a similar manner the $\left\{y_{i}\right\}$ determine the distance between the horizontal lines. Then the probability that the edge between two adjacent vertices is open is $p^{d}$, where $d$ is the distance between the vertices. All edges are open independently of each other. This generates the same percolation process as was described above.

For each $p$ this defines a measure $P_{p}$ on $\Omega=\{0,1\}^{E\left(\mathbb{Z}^{2}\right)}$. Let $\omega \in \Omega$ be a realization of percolation. An open cluster $C$ for $\omega$ is a maximal connected subset of edges such that all edges $e \in C$ are open $(\omega(e)=1$ for all $e \in C)$. Jonasson, Mossel and Peres were able to show that a similar process on $\mathbb{Z}^{3}$ exhibits a phase transition [6]. That is they proved the following theorem.

Theorem 1.1 [6] For percolation on the randomly stretched lattice in $\mathbb{Z}^{3}$ there exists a value $p_{c}, 0<p_{c}<1$ such that if $p<p_{c}$ then

$$
P_{p}(\exists \text { an infinite open cluster })=0 .
$$

If $p>p_{c}$ then

$$
P_{p}(\exists \text { an infinite open cluster })=1 .
$$


In this paper we establish that percolation on a randomly stretched lattice on $\mathbb{Z}^{2}$ also exhibits a phase transition. The proof of Jonasson, Mossel and Peres works to show that for the randomly stretched lattice in $\mathbb{Z}^{2}$ with $p$ close to zero that there is no infinite cluster a.s. Thus we need only to show that for $p$ close to one that there is an infinite cluster a.s.

Before we do that we introduce a different model of dependent percolation which we call percolation on a regularly stretched lattice. This model will also exhibit a phase transition. We introduce this model because the proof of a phase transition is much simpler than on the randomly stretched lattice, yet it contains the essential ideas of the proof of a phase transition on the randomly stretched lattice.

The only difference between these two models is the choice of the $\left\{x_{i}\right\}_{i \in \mathbb{Z}}$ and $\left\{y_{i}\right\}_{i \in \mathbb{Z}}$. Instead of random variables as they were for the randomly stretched lattice they will be deterministic. Let $x_{0}=y_{0}=1$. Given $i$ let $x_{i}=y_{i}=q$ if $4^{q-1} \mid i$ but $4^{q} \nmid i$. Then the edges are open or closed independently with the probabilities listed above.

\section{Phase transition on the regularly stretched lattice}

In this section we show that the regularly stretched lattice exhibits a phase transition. First we show that for small $p$ there is no infinite connected cluster. The proof is identical to the proof in [6] that percolation on a randomly stretched lattice does not have an infinite cluster when $p$ is small.

Theorem 2.1 On the regularly stretched lattice if $p<\frac{1}{4}$ then

$$
P_{p}(\exists \text { an infinite connected cluster })=0 .
$$

Proof. For any $j$ let

$$
\begin{aligned}
\delta_{j}= & \left.\left\{\left( \pm 4^{j}, c\right),\left( \pm 4^{j}+1, c\right)\right),|c| \leq 4^{j}\right\} \bigcup \\
& \left\{\left(\left(d, \pm 4^{j}\right),\left(d, \pm 4^{j}+1\right)\right),|d| \leq 4^{j}\right\} .
\end{aligned}
$$

This group of edges separates a square containing 0 from infinity. Each edge in $\delta_{j}$ has length $j$. Then

$$
\begin{aligned}
P_{p}\left(\exists \text { an open edge in } \delta_{j}\right) & \leq 4\left(2 \cdot 4^{j}+1\right) p^{j} \\
& \leq 16(4 p)^{j} .
\end{aligned}
$$

Now suppose the vertex $(a, b)$ is in an infinite cluster. Then if $4^{j}>|a|,|b|$ then there exists an open edge in $\delta_{j}$. Thus by Borel Cantelli if $p<1 / 4$ then

$$
P_{p}(\exists \text { an infinite connected cluster })=0 .
$$


To show that when $p$ is close to 1 that there is an infinite cluster we use an argument reminiscent of Chayes, Chayes, and Durrett's proof that there exists crossings of a square in certain examples of fractal percolation [2].

First we define $B_{n}(a, b)$ to be the set of vertices $(i, j)$ such that $a<i \leq a+4^{n-1}$ and $b<j \leq b+4^{n-1}$. An $\mathbf{n}$ box is a graph that has vertices $B_{n}(a, b)$ where $4^{n} \mid a$ and $4^{n} \mid b$. It consists of all edges between two vertices in $B_{n}(a, b)$. If $a \neq 0$ and $b \neq 0$ and $B_{n}(a, b)$ is an $n$ box then all the edges emanating from $B_{n}(a, b)$ have length $n$ in the regularly stretch lattice.

Now we will inductively define what it means for an $n$ box to be good. Let $\omega$ be a realization of percolation. A 1 box is good if every edge in the 1 box is open. Assume we have defined a good $n-1$ box in such a way that if an $n-1$ box is good then there exists a unique cluster which intersects all four sides of the $n-1$ box. This is called the crossing cluster. An $n$ box is good if

1. at least 15 of the $16 n-1$ boxes inside the $n$ box are good and

2. between each pair of adjacent $n-1$ boxes which are good there exists an open edge which connects the crossing clusters of the good $n-1$ boxes.

It is easy to check that a good $n$ box also contains a crossing cluster.

Lemma 2.1 Every good $n$ box has a crossing cluster.

Proof. This follows easily by induction. The crossing cluster of the good $n$ box contains the union of the crossing clusters of the good $n-1$ boxes.

The key idea in the proof of the phase transition for the regularly stretched lattice is the following lemma.

Lemma 2.2 Let $B_{n}(a, b)$ and $B_{n}\left(a+4^{n}, b\right)$ be good $n$ boxes. There are at least $2^{n}$ edges which intersect the crossing clusters of both $B_{n}(a, b)$ and $B_{n}\left(a+4^{n}, b\right)$.

Proof. Each pair of adjacent $n$ boxes, $B_{n}(a, b)$ and $B_{n}\left(a+4^{n}, b\right)$, contains four pairs of adjacent $n-1$ boxes, $B_{n}\left(a+3 \cdot 4^{n-1}, b+i 4^{n-1}\right)$ and $B_{n}\left(a+4^{n}, b+i 4^{n-1}\right)$ for $i=0,1,2$, 3 . If both $B_{n}(a, b)$ and $B_{n}\left(a+4^{n}, b\right)$ are good then at least six of the above $n-1$ boxes are good. Thus for at least two of the above pairs both of the adjacent $n-1$ boxes are good. By the induction hypothesis each of these pairs has at least $2^{n-1}$ edges which connect the crossing clusters of the $n-1$ boxes and thus connect the crossing clusters of the $n$ boxes. Thus there are at least $2^{n}$ edges which connect the crossing clusters of $B_{n}(a, b)$ and $B_{n}\left(a+4^{n}, b\right)$.

Lemma 2.3 If $p>1-2^{-1000}$ then for any $n$ box $B_{n}(a, b)$

$$
P_{p}\left(B_{n}(a, b) \text { is good }\right) \geq 1-\frac{1}{1000} e^{-n} \text {. }
$$


Proof. The proof is by induction. The lemma is clearly true when $n=10$. The probability that the first condition for any $n$ box to be not good is less than

$$
\left(\begin{array}{c}
16 \\
2
\end{array}\right) P_{p}\left(B_{n-1}(a, b) \text { is not good }\right)^{2} \leq 120\left(\frac{1}{1000}\right)^{2} e^{-2 n+2} \leq .5\left(\frac{1}{1000} e^{-n}\right) .
$$

If $B_{n-1}(a, b)$ and $B_{n-1}(c, d)$ are neighboring $n-1$ boxes which are both good then there must be at least $2^{n}$ pairs of vertices, one in each of the crossing clusters, which are separated by one edge. This is true by Lemma 2.2. Each of these edges is open with probability $p^{n}$. The probability that all $2^{n}$ are closed is

$$
\left(1-p^{n}\right)^{2^{n}} \leq 2^{-\left(2^{n} p^{n}\right)} \leq 2^{-1.5^{n}}
$$

There are $\left(\begin{array}{c}16 \\ 2\end{array}\right)$ pairs of adjacent $n-1$ boxes in an $n$ box. Thus the probability that condition 2 is not satisfied is less than $\left(\begin{array}{c}16 \\ 2\end{array}\right) 2^{-1.5^{n}}$. Thus

$$
P\left(B_{n}(a, b) \text { is good }\right) \geq 1-.5\left(\frac{1}{1000} e^{-n}\right)-\left(\begin{array}{c}
16 \\
2
\end{array}\right) 2^{-1.5^{n}} \geq 1-\frac{1}{1000} e^{-n} .
$$

From this it is easy to conclude that there is an infinite open cluster.

Theorem 2.2 If $p>1-2^{-1000}$ then

$$
P_{p}(\exists \text { an infinite open cluster })=1 \text {. }
$$

Proof. There exists some $N$ such that $B_{n}(0,0)$ is good for all $n \geq N$ a.s. Thus there is some vertex $(i, j)$ which is contained in a good $n$ box for all $n$. Thus it is in the crossing cluster for all $n$ and is connected to an infinite number of other vertices.

\section{Creating a regular lattice from a random lattice}

In trying to adapt the proof in the previous section to the case of the randomly stretched lattice we have two main obstacles to overcome. First we must figure out how to fit a regular lattice onto the random $\left\{x_{i}\right\}$ and $\left\{y_{i}\right\}$. That is the subject of this section. Then in the next section we check to see that the proof still applies to the lattice structure that we imposed.

Each integer $y$ defines a row which is the set of all edges that connect $(x, y)$ to $(x, y+1)$ for some $x$. Each integer $x$ defines a column which is the set of all edges that connect $(x, y)$ to $(x+1, y)$ for some $y$. In the regularly stretched lattice there were rows and columns which it was hard for the percolation cluster to get through. On the randomly stretched lattice we will group neighboring rows and columns into bands. In the regular lattice the row generated by $i$ was assigned the number $y_{i}$. In this model each band will be assigned a number. A higher number indicates that that there are fewer paths across the band. We will assign the 
rows (and columns) into bands in such a way that if two bands are both assigned a high number then they must be exponentially far apart. This will allow us to mimic the proof in the previous section.

Starting with $\left\{x_{i}\right\}$ we will construct a sequence of functions $f_{l}: \mathbb{Z} \rightarrow \mathbb{N}$. An $l$ band is a collection of consecutive integers $[i, j]$. A necessary condition for $[i, j]$ to be an $l$ band is that

$$
f_{l}(i)=f_{l}(i+1)=\cdots=f_{l}(j) .
$$

A band is an interval of integers which is an $l$ band for all $l$ greater than some $L$.

Each $i \in \mathbb{Z}$ is a 1 band. We assign the 1 bands labels by $f_{1}(i)=\left\lceil x_{i}\right\rceil$, the smallest integer greater than or equal to $x_{i}$. We also use the notation $\left\lfloor x_{i}\right\rfloor$ for the largest integer less than or equal to $x_{i}$.

We now begin the inductive procedure in which we combine multiple $n$ bands into a single $n+1$ band and assign this band a higher number. Find $i$ such that $|i+.1|$ is the smallest for which there exists $j$, with the following properties

1. $j \neq i$,

2. $|j| \leq|i|$, and

3. $\min \left(f_{1}(i), f_{1}(j)\right)-\frac{1}{6} \log _{2}|i-j|>1$.

The 2 bands then consist of the interval $[j, i]$ (or $[i, j]$ if $i<j$ ) and every integer $k \notin[j, i]$. We set

$$
f_{2}(k)= \begin{cases}f_{1}(k) & k \notin[j, i] \\ f_{1}(i)+f_{1}(j)-\left\lfloor\frac{1}{18} \log _{2}|i-j|\right\rfloor & k \in[j, i] .\end{cases}
$$

Now assume that $n$ bands and $f_{n}$ have been defined. Let $i$ be the integer such that $|i+.1|$ is the smallest for which there exist a $j$ with the following properties

1. $j$ is not in the same $n$ band as $i$,

2. $|j| \leq|i|$, and

3. $\min \left(f_{n}(i), f_{n}(j)\right)-\frac{1}{6} \log _{2}(1+\#$ of $n$ bands between $i$ and $j)>1$.

Then the $n+1$ bands are the union of the $n$ bands intersecting the interval $[i, j]$ and every $n$ band not intersecting the interval $[i, j]$. We set

$$
f_{n+1}(k)= \begin{cases}f_{n}(k) & n \text { band of } k \cap[i, j]=\emptyset \\ f_{n}(i)+f_{n}(j)-\left\lfloor\frac{1}{18} \log _{2}(1+\# \text { of } n \text { bands in }(i, j))\right\rfloor & n \text { band of } k \cap[i, j] \neq \emptyset\end{cases}
$$

Let $f(k)=\lim _{n \rightarrow \infty} f_{n}(k)$ and let a band be an interval of integers for which there is an $N$ such that the interval was a $n$ band for all $n>N$.

We will now work towards proving that $f(k)$ exists and is finite for all $k$ a.s.

Lemma 3.1 If $[j, k]$ is an $n$ band and $f_{n}(j)=m$ then $|k-j+1| \leq 32^{m-1}$. 
Proof. The proof is by induction on $m$. The statement is true for $m=1$ and $m=2$ because for these we must have $j=k$. Now suppose $[j, k]$ is an $n$ band, $j \neq k$, and $f_{n}(j)=m>2$. Then there exists some $n^{\prime}$ such that $[j, k]$ is an $n^{\prime}$ band but $[j, k]$ is not an $n^{\prime}-1$ band. Let $j^{\prime}, k^{\prime}, p$, and $q$ be defined so that $\left[j, j^{\prime}\right]$ and $\left[k^{\prime}, k\right]$ are $n^{\prime}-1$ bands, $p=f_{n^{\prime}-1}(j)$ and $q=f_{n^{\prime}-1}(k)$. Then $p, q<m$ so the induction hypothesis holds for all $n^{\prime}-1$ bands in $[j, k]$. For any $l<\min (p, q)$ there are at least $64^{l-1}-1 n^{\prime}-1$ bands in between any two $n^{\prime}-1$ bands in $[j, k]$ each with labels $\geq l$. Let

$$
N=\text { the number of } n^{\prime}-1 \text { bands between }\left[j, j^{\prime}\right] \text { and }\left[k^{\prime}, k\right] \text {. }
$$

Then there are at most $N / 64^{l-1} n^{\prime}-1$ bands with labels $l$ between $\left[j, j^{\prime}\right]$ and $\left[k^{\prime}, k\right]$. Thus

$$
\begin{aligned}
|k-j+1| & =\left|k-k^{\prime}+\left(k^{\prime}-j^{\prime}\right)+\left(j^{\prime}-j\right)+1\right| \\
& \leq\left|k-k^{\prime}+1\right|+\left|j^{\prime}-j+1\right|+\sum_{n^{\prime}-1 \text { bands in }\left(j^{\prime}, k^{\prime}\right)}\left|b^{\prime}-b+1\right| \\
& \leq\left|k-k^{\prime}+1\right|+\left|j^{\prime}-j+1\right|+\sum_{l} \sum_{n^{\prime}-1 \text { bands in }\left(j^{\prime}, k^{\prime}\right) \text { with label } l}\left|b^{\prime}-b+1\right| \\
& \leq 32^{q-1}+32^{p-1}+\sum_{l}\left(N / 64^{l-1}\right) 32^{l-1} \\
& \leq 32^{q-1}+32^{p-1}+2 N \\
& \leq 2 \cdot 32^{\max (p, q)-1}+2^{6 \min (p, q)-5} \\
& \leq \frac{1}{16} 32^{m-1}+2^{6 \min (p, q)-5} \\
& \leq \frac{1}{16} 32^{m-1}+\frac{1}{2} 2^{(15 / 2) \min (p, q)-5} \\
& \leq \frac{1}{16} 32^{m-1}+\frac{1}{2} 32^{(3 / 2) \min (p, q)-1} \\
& \leq \frac{1}{16} 32^{m-1}+\frac{1}{2} 32^{m-1} \\
& \leq 32^{m-1} .
\end{aligned}
$$

Line 1 is true because $\min \left(f_{n^{\prime}-1}(j), f_{n^{\prime}-1}(k)\right)-\frac{1}{6} \log _{2}(1+N)>1$. Line 2 is true because $m \geq$ $\max (p, q)+1$. Line 3 is true because $\min (p, q) \geq 2$. Line 4 is true because $m \geq \frac{3}{2} \min (p, q)$.

Lemma 3.2 If $\left[j, j^{\prime}\right]$ and $\left[k^{\prime}, k\right]$ are two $n$ bands which are combined to form an $n+1$ band $[j, k]$ then

$$
N=\text { the number of } n \text { bands in }\left(j^{\prime}, k^{\prime}\right) \geq\left(k^{\prime}-j^{\prime}-1\right) / 2 \text {. }
$$

Proof. Let $N_{l}$ be the number of $n$ bands in $\left(j^{\prime}, k^{\prime}\right)$ with labels $l$. Then $N=\sum N_{l}$. Also $N_{l} \leq N / 64^{l-1}$ because if not then there would have been two other $n$ bands which would 
have combined.

$$
\begin{aligned}
k^{\prime}-j^{\prime}-1 & =\sum_{\left[b, b^{\prime}\right] \text { a band in }\left(j^{\prime}, k^{\prime}\right)}\left(b^{\prime}-b+1\right) \\
& \leq N_{1}+N_{2}+\sum_{l \geq 3}\left(N / 64^{l-1}\right) 32^{l-1} \\
& \leq N_{1}+N_{2}+N / 2 \\
& \leq 2 N
\end{aligned}
$$

so $N \geq\left(k^{\prime}-j^{\prime}-1\right) / 2$.

Before we proceed we must introduce some more notation. If the interval $[j, k]$ is an $n$ band then we say $j$ and $k$ are the $n$ generators of the $n$ band. The $n-1$ generators of the $n$ band $[j, k]$ are the $n-1$ generators of the $n-1$ bands containing $j$ and $k$. Suppose for all $n$, all $n$ bands and some $i$ the $n-i$ generators of an $n$ band have been defined. Then for any $n$ band define the $n-(i+1)$ generators of the $n$ band as the union of the $n-(i+1)$ generators of the $n-(i+1)$ bands which contain an $(n-i)$ generator of the $n$ band. We call the 1 generators of an $n$ band the generators of an $n$ band.

We say that a generator $g$ of a band $[i, j]$ is a maximal generator of $[i, j]$ if the following condition is satisfied. For each pair of $k$ bands $\left[i_{1}, j_{1}\right]$ and $\left[i_{2}, j_{2}\right]$ which form $k+1$ band $\left[i_{1}, j_{2}\right]$ such that $g \in\left[i_{1}, j_{2}\right]$, the label of the $k$ band that contains $g$ is at least as big as the label of the $k$ band that doesn't contain $g$.

Lemma 3.3 Let $i_{1}<i_{2}<\cdots<i_{k}$ be the generators of an $n$ band with $\sum_{1}^{k} f_{1}\left(i_{j}\right)=m$. Then

$$
\sum_{2}^{k}\left\lfloor\log _{2}\left(i_{j}-i_{j-1}+1\right)\right\rfloor \leq 37 m .
$$

Proof. For each $j, 1<j \leq k$, let $n_{j}$ be the value such that there exists $a$ and $b$ so $n_{j}$ bands $\left[i_{a}, i_{j-1}\right]$ and $\left[i_{j}, i_{b}\right]$ are merged into $n_{j+1}$ band $\left[i_{a}, i_{b}\right]$. Let $q_{j}$ be the number of $n_{j}$ bands between $\left[i_{a}, i_{j-1}\right]$ and $\left[i_{j}, i_{b}\right]$. By the previous lemma

$$
\frac{1}{2}\left(i_{j}-i_{j-1}+1\right) \leq 1+q_{j} .
$$

By the assumption that $\sum_{1}^{k} f_{1}\left(i_{j}\right)=m$ we have that

$$
\sum_{j=2}^{k}\left\lfloor\frac{1}{18} \log _{2}\left(1+q_{j}\right)\right\rfloor \leq m .
$$


Thus

$$
\begin{aligned}
\sum_{2}^{k}\left\lfloor\frac{1}{18} \log _{2}\left(\frac{1}{2}\left(i_{j}-i_{j-1}+1\right)\right)\right\rfloor & \leq m \\
\sum_{2}^{k}\left(\frac{1}{18}\left\lfloor\log _{2}\left(\frac{1}{2}\left(i_{j}-i_{j-1}+1\right)\right)\right\rfloor-1\right) & \leq m \\
\sum_{2}^{k}\left\lfloor\log _{2}\left(\frac{1}{2}\left(i_{j}-i_{j-1}+1\right)\right)\right\rfloor & \leq 36 m \\
\sum_{2}^{k}\left\lfloor\log _{2}\left(i_{j}-i_{j-1}+1\right)\right\rfloor & \leq 37 m
\end{aligned}
$$

Lemma 3.4 For any $j$ and $l$

$P(\exists n$ such that $j$ is contained in an $n$ band with label $\geq l) \leq 2^{-399 l}$.

Proof. If there exists an $n$ and $l$ then $\exists$ generators $i_{1}, \ldots, i_{k}$ and $m$ such that

$$
\sum_{k} f_{1}\left(i_{k}\right)=m \geq l
$$

By Lemma 3.1 we have that $i_{1} \leq j \leq i_{1}+32^{l-1}-1$. By Lemma 3.3 there are at most $2^{37 m}$ choices of $\left\lfloor\log _{2}\left(i_{2}-i_{1}+1\right)\right\rfloor, \ldots,\left\lfloor\log _{2}\left(i_{k}-i_{k-1}+1\right)\right\rfloor$. Given a choice of $\left\lfloor\log _{2}\left(i_{2}-i_{1}+\right.\right.$ $1)\rfloor, \ldots,\left\lfloor\log _{2}\left(i_{k}-i_{k-1}+1\right)\right\rfloor$ there are at most $2^{37 m}$ choices of $\left(i_{2}-i_{1}\right), \ldots,\left(i_{k}-i_{k-1}\right)$. Thus there are at most $32^{l-1}\left(2^{74 m}\right) \leq 2^{79 m}$ choices of $i_{1}, \ldots, i_{k}$. For each choice of $i_{1}, \ldots, i_{k}$ there are at most $2^{m}$ choices for $f_{1}\left(i_{1}\right), \ldots, f_{1}\left(i_{k}\right)$. Thus there are at most $2^{80 m}$ choices of $i_{1}, \ldots, i_{k}$ and $f_{1}\left(i_{1}\right), \ldots, f_{1}\left(i_{k}\right)$. Each choice has probability $\leq 2^{-1000(m-k)} \leq 2^{-500 m}$. Thus

$$
\begin{aligned}
P(\exists n \text { such that } j \text { is contained in an } n \text { band with labels } \geq l) & \leq \sum_{m \geq l} 2^{-400 m} \\
& \leq 2^{-399 l} .
\end{aligned}
$$

Lemma 3.5 For each $j \in \mathbb{Z}$ there exists $N$ such that for all $n>N$ the $n$ band containing $j$ is the same a.s.

Proof. This follows easily from Lemma 3.4 and Borel-Cantelli.

We say that two $(n)$ bands $\left[i_{1}, j_{1}\right]$ and $\left[i_{2}, j_{2}\right]$ with labels $\geq l$ are neighboring $(n)$ bands with labels $\geq l$ if there exists no $(n)$ band $\left[i_{3}, j_{3}\right]$ with $j_{1}<i_{3} \leq j_{3}<i_{2}$ with label $\geq l$. 
Lemma 3.6 Between any two neighboring bands with labels $\geq l$ there exists at least $64^{l-1}-1$ other bands.

Proof. If not then the bands would have been combined at some level.

We call a sequence $\left\{x_{i}\right\}$ regular if the procedure above generates bands such that for all $l$ and all neighboring bands with labels greater than or equal to $l,\left[i_{1}, j_{1}\right]$ and $\left[i_{2}, j_{2}\right]$, the number of bands in $\left(j_{1}, j_{2}\right)$ is in the interval $\left[64^{l-1}-1,6 \cdot 64^{l-1}\right)$. It is easy to check that if $\left\{x_{i}\right\}$ is regular then for any $l$ and between any two bands neighboring bands with labels $\geq l$ there exists between 10 and 383 bands with labels $l-1$.

Lemma 3.7 For almost every sequence $\left\{x_{i}\right\}$ there exists a sequence $\left\{\widetilde{x}_{i}\right\}$ such that

1) $\widetilde{x}_{i} \geq x_{i}$ for all $i$,

2) the $k$ bands for $\left\{\widetilde{x}_{i}\right\}$ are the same as the $k$ bands for $\left\{x_{i}\right\}$ for all $k$, and

3) $\left\{\widetilde{x}_{i}\right\}$ is regular.

Proof. First, for any $L$, we prove the statement with condition 3) replaced by

$\left.3^{\prime}\right)$ for all $l \leq L$ and neighboring bands with labels $\geq l,\left[i_{1}, j_{1}\right]$ and $\left[i_{2}, j_{2}\right]$, the number of bands in $\left(j_{1}, j_{2}\right)$ is in the interval $\left[64^{l-1}-1,3 \cdot 64^{l-1}\right)$.

We do this by induction on $L$. It is trivially true for $L=1$. Suppose $\left\{x_{i}^{L}\right\}$ is a sequence with this property for $L$. We will construct a sequence $\left\{x_{i}^{L+1}\right\}$ with this property for $L+1$.

Let $\left[i_{1}, j_{1}\right]$ and $\left[i_{2}, j_{2}\right]$ be neighboring bands with labels $\geq L+1$. Also let

$$
N=\text { the number of bands in }\left(j_{1}, i_{2}\right) \text {. }
$$

We will show that either $64^{L}-1 \leq N<3 \cdot 64^{L}$ or it is possible to pick one $i \in\left(j_{1}, j_{2}\right)$ and to increase one $x_{i}^{L}$ to $x_{i}^{L}+1$. This produces a new sequence with the same $k$ bands for all $k$, the label of the band containing $i$ is increased by one. From this it is easy to conclude that the modified version of the lemma is true.

We have that $N \geq 64^{L}-1$ because, if it were not, then the bands $\left[i_{1}, j_{1}\right]$ and $\left[i_{2}, j_{2}\right]$ would have been combined into a single band. Thus we only need to consider the case where $N \geq 3 \cdot 64^{L}$. In this case, by the induction hypothesis, there exists a band $\left[i_{3}, j_{3}\right]$ with label $L$ and $j_{1}<i_{3}<i_{2}$. Furthermore we can demand the number of bands in $\left(j_{2}, i_{3}\right)$ is in the interval $\left[64^{L}-1,64^{L}+3 \cdot 64^{L-1}\right]$. Thus the number of bands in $\left(j_{3}, i_{2}\right)$ is at least

$$
3 \cdot 64^{L}-\left(64^{L}+3 \cdot 64^{L-1}+1\right)>64^{L}-1 .
$$

Choose $i^{\prime} \in\left[i_{3}, j_{3}\right]$ to be a maximal generator of $\left[i_{3}, j_{3}\right]$. Setting

$$
\bar{x}_{i}= \begin{cases}x_{i}^{L} & i \neq i^{\prime} \\ x_{i}^{L}+1 & i=i^{\prime} .\end{cases}
$$


Inductively it is true that for all $k$, the $k$ bands for $\left\{x_{i}^{L}\right\}$ and $\left\{\bar{x}_{i}\right\}$ are the same, the labels of any $k$ bands for $\left\{x_{i}^{L}\right\}$ and $\left\{\bar{x}_{i}\right\}$ that do not contain $i^{\prime}$ are the same, and the labels for the $k$ bands for $\left\{x_{i}^{L}\right\}$ that contain $i^{\prime}$ are one less than the labels of the same $k$ bands for $\left\{\bar{x}_{i}\right\}$. Thus we can create $\left\{x_{i}^{L+1}\right\}$.

By the above procedure we get an increasing (in $L$ ) sequence of sequences $\left\{x_{i}^{L}\right\}$. It is clear that $\lim _{L} x_{i}^{L}$ exists for all $i$ except possibly one band. Call that band $\left[i_{4}, j_{4}\right]$. Set

$$
\widetilde{x}_{i}= \begin{cases}\lim _{L} x_{i}^{L} & i \notin\left[i_{4}, j_{4}\right] \\ x_{i} & i \in\left[i_{4}, j_{4}\right] .\end{cases}
$$

Then this sequence has the property that between any two neighboring bands with labels $\geq l$ the number of bands in between is in the interval $\left[64^{l-1}, 6 \cdot 64^{l-1}\right)$. Such a sequence is regular and the lemma is true.

When we defined what it means for a sequence to be regular we were concerned with neighboring bands with labels $\geq l$ being too far apart. We now define what it means for a sequence to be very regular. In addition to the bands being regular we demand a regularity condition of the $k$ bands that are combined in order to form a $k+1$ band.

A vertical band is a band that is generated from the $\left\{x_{i}\right\}$. A horizontal band is a band that is generated from the $\left\{y_{i}\right\}$. Suppose $\left[i_{1}, j_{1}\right]$ and $\left[i_{2}, j_{2}\right]$ are neighboring horizontal bands with label $\geq n$ and $\left[i_{3}, j_{3}\right]$ and $\left[i_{4}, j_{4}\right]$ are neighboring vertical bands with label $\geq n$. Then the graph with vertices

$$
V=\left\{(x, y): j_{3}+1 \leq x \leq i_{4}, j_{1}+1 \leq y \leq i_{2}\right\}
$$

and edges

$$
E=\{\text { edges between two vertices in } V \text { with at most one edge in } \delta V\}
$$

is called an $n$ box. We write $\left[j_{3}+1, i_{4}\right] \times\left[j_{1}+1, i_{2}\right]$ is an $n$ box. We will also call any subgraph $\left[j_{3}+1, i_{4}\right] \times\left[j_{1}+1, i_{2}\right]$ an $n$ box if there exists $\left\{\bar{x}_{i}\right\}$ and $\left\{\bar{y}_{i}\right\}$ which are regular such that

$$
\begin{aligned}
& \bar{x}_{m}=x_{m} \text { for all } m \text { such that } j_{3}<m \leq i_{4}, \\
& \bar{y}_{m}=y_{m} \text { for all } m \text { such that } j_{1}<m \leq i_{2},
\end{aligned}
$$

and $\left[j_{3}+1, i_{4}\right] \times\left[j_{1}+1, i_{2}\right]$ is an $n$ box (for $\left\{\bar{x}_{i}\right\}$ and $\left\{\bar{y}_{i}\right\}$ ).

Suppose $\left[i_{5}, j_{5}\right]$ is a horizontal (vertical) band with label $n$ and $\left[j_{3}+1, i_{4}\right] \times\left[j_{1}+1, i_{2}\right]$ is an $m$ box. Then we say $\left(j_{3}, i_{4}\right] \times\left[i_{5}, j_{5}+1\right]\left(\left[i_{5}, j_{5}+1\right] \times\left[j_{1}+1, i_{2}\right]\right)$ is a horizontal (vertical) $(m, n)$ strip. This graph has vertices

$$
V=\left\{(x, y): j_{3}+1 \leq x \leq i_{4}, i_{5} \leq y \leq j_{5}+1\right\}
$$

and edges

$$
E=\{\text { edges between two vertices in } V \text { with at most one edge in } \delta V\} .
$$


We will also call any subgraph $\left[j_{3}+1, i_{4}\right] \times\left[i_{5}, j_{5}+1\right]$ an $(m, n)$ strip if there exists $\left\{\bar{x}_{i}\right\}$ and $\left\{\bar{y}_{i}\right\}$ which are regular such that

$$
\begin{aligned}
& \bar{x}_{m}=x_{m} \text { for all } m \text { such that } j_{3}<m \leq i_{4}, \\
& \bar{y}_{m}=y_{m} \text { for all } m \text { such that } i_{5}<m \leq j_{5},
\end{aligned}
$$

and $\left[j_{3}+1, i_{4}\right] \times\left[i_{5}, j_{5}+1\right]$ is an $(m, n)$ strip (for $\left\{\bar{x}_{i}\right\}$ and $\left\{\bar{y}_{i}\right\}$ ).

Any $k$ band of the form $[a, a]$ is very regular. Any 1 box is very regular. Any 2 box $[a, b] \times[c, d]$ is very regular if $11 \leq b-a \leq 384$ and $11 \leq d-c \leq 384$. We now inductively define what it means for a $k$ band with label $l$ to be very regular and for an $n$ box to be very regular. Let $[a, b]$ be a $k$ band with label $l$ which was formed by combining $k-j$ band $\left[a, a^{\prime}\right]$ and $\left[b^{\prime}, b\right]$ into $k-j+1$ band $[a, b]$. We say that $[a, b]$ is very regular if there exists $m \leq 384$, $c_{1}=a^{\prime}, c_{2}, c_{3}, \ldots, c_{m}, d_{1}, d_{2}, \ldots, d_{m}=b^{\prime}$, and $q$ such that

1) all $k-j$ bands in $[a, b]$ are very regular $k-j$ bands

2) $\left[c_{t}, d_{t}\right] \times\left[c_{t}, d_{t}\right]$ in $\left\{x_{i}\right\} \times\left\{x_{i}\right\}$ is a very regular $q$ box for all $t$, and

3) $\left[d_{t}, c_{t+1}-1\right]$ is a very regular $k-j$ band with label $q$.

An $l$ box is very regular if

1) $\left\{x_{i}\right\}$ and $\left\{y_{i}\right\}$ are regular and

2) all bands with labels $l-1$ and $l-1$ boxes inside the $l$ box are very regular.

We say a sequence $\left\{x_{i}\right\}$ is very regular if all the bands generated by $\left\{x_{i}\right\}$ are very regular.

\section{Lemma 3.8 If}

1) $[a, b]$ is a $k$ band with label $l$

2) $a^{\prime}$ is a maximal generator of $[a, b]$

3) the label of the $j$ band containing $[a, b]$ is $l_{j}$

4) for all $j>k$ such that $a^{\prime}$ is a generator of the $j$ band containing $[a, b]$ the $64^{l_{j}} j$ bands on either side of the band containing $[a, b]$ have label $<l_{j}+1$

and we set

$$
\bar{x}_{i}= \begin{cases}x_{i} & \text { if } i \neq a^{\prime} \\ x_{a^{\prime}}+1 & \text { if } i=a^{\prime}\end{cases}
$$

then we have

i) the $j$ bands of $\left\{x_{i}\right\}$ and the $j$ bands of $\left\{\bar{x}_{i}\right\}$ are the same for all $j$

ii) for all $j$ bands $\left[e_{j}, f_{j}\right]$ of $\left\{\bar{x}_{i}\right\}$ which has $a^{\prime}$ as a generator has label $l_{j}+1$ and 
iii) for all $j$ bands of $\left\{\bar{x}_{i}\right\}$ that do not have $a^{\prime}$ as a generator have the same label as for $\left\{x_{i}\right\}$.

Proof. We prove the lemma by induction on $j$. It is clearly true for $j=1$. We assume that it is proven for $j$. By condition 4 and ii) and iii) for $j$ we have that i) holds for $j+1$. As the $j$ bands are the same ii) and iii) for $j$ imply that ii) and iii) hold for $j+1$.

Lemma 3.9 If in addition the hypotheses in Lemma 3.8 we add

5) $[a, b]$ is very regular (for $\left.\left\{x_{i}\right\}\right)$

then we get the additional conclusion

iv) $[a, b]$ is very regular (for $\left.\left\{\bar{x}_{i}\right\}\right)$.

Proof. We can prove the lemma by proving that for any $j$ all $j$ bands in $[a, b]$ are very regular. We do this by induction. It is clearly true for $j=1$. Assume it is proven for $j$. All the $j$ bands are very regular since $[a, b]$ is very regular (for $\left\{x_{i}\right\}$ ). All the other conditions are also satisfied since $[a, b]$ is very regular (for $\left\{x_{i}\right\}$ ). Thus the $j+1$ bands are regular (for $\left.\left\{\bar{x}_{i}\right\}\right)$.

Lemma 3.10 For almost every sequence $\left\{x_{i}\right\}$ there exists a sequence $\left\{\widehat{x}_{i}\right\}$ such that

1) $\widehat{x}_{i} \geq x_{i}$ for all $i$ and

2) $\left\{\widehat{x}_{i}\right\}$ is very regular.

Proof. By Lemma 3.7 we may assume that $\left\{x_{i}\right\}$ is regular. We construct sequences $\left\{x_{i}^{j}\right\}$ such that $\left\{x_{i}^{j}\right\}$ is nondecreasing as $j$ increases. The $k$ bands of $\left\{x_{i}^{j}\right\}$ are the same as the $k$ bands of $\left\{x_{i}\right\}$ for all $j$ and $k$. Also the $k$ bands of $\left\{x_{i}^{j}\right\}$ are very regular for all $k \leq j$. We do this inductively. It can be done for $j=1$. Suppose it can be done for $j$. Then there is at most one $j+1$ band $[a, b]$ which is not a $j$ band. Hence there is at most one $j+1$ band which is not very regular (for $\left\{x_{i}^{j}\right\}$ ).

We now employ the method in Lemma 3.7 as well as Lemma 3.9 to make $[a, b]$ very regular for $\left\{x_{i}^{j+1}\right\}$. We show inductively for any $L$ and all $l \leq L$ and neighboring $j$ bands in $[a, b]$ with labels $\geq l$ (call them $\left[i_{1}, j_{1}\right]$ and $\left[i_{2}, j_{2}\right]$ ) the number of $j$ bands in $\left(j_{1}, i_{2}\right)$ is in the interval $\left[64^{l-1}, 3 \cdot 64^{l-1}\right)$. It is trivial for $L=1$.

Let $\left[i_{1}, j_{1}\right]$ and $\left[i_{2}, j_{2}\right]$ be neighboring $j$ bands with labels $\geq L+1$. Also let

$$
N=\text { the number of } j \text { bands in }\left(j_{1}, i_{2}\right) \text {. }
$$

We have that $N \geq 64^{L}$ because if it were not then the $j$ bands $\left[i_{1}, j_{1}\right]$ and $\left[i_{2}, j_{2}\right]$ would be combined into a single $j+1$ band for $\left\{x_{i}^{j}\right\}$. If $N<3 \cdot 64^{L}$ we do nothing. If $N \geq 3 \cdot 64^{L}$ there exists a $j$ band $\left[i_{3}, j_{3}\right]$ which is very regular, has label $L$ and $j_{1}<i_{3}<i_{2}$. Furthermore we 
can demand that the number of $k$ bands in $\left(j_{2}, i_{3}\right)$ is in the interval $\left[64^{L}-1,64^{L}+3 \cdot 64^{L-1}\right)$. Thus the number of $k$ bands in $\left(j_{3}, i_{2}\right)$ is at least $3 \cdot 64^{L}-\left(64^{L}+3 \cdot 64^{L-1}\right)>64^{L}$.

Let $i^{\prime}$ be a maximal generator of $\left[i_{3}, j_{3}\right]$. Set

$$
\bar{x}_{i}= \begin{cases}x_{i}^{j}+1 & i=i^{\prime} \\ x_{i}^{j} & i \neq i^{\prime} .\end{cases}
$$

Then by Lemma 3.9 the $m$ bands of $\left\{\bar{x}_{i}\right\}$ are the same as the $m$ bands as $\left\{x_{i}^{j}\right\}$ for all $m$. The $j$ band $\left[i_{3}, j_{3}\right]$ has label $L+1$. Continuing in this manner we can show that the induction hypothesis is true for $L+1$. By repeating this procedure we construct $\left\{x_{i}^{j}\right\}$. Then set $\widetilde{x}_{i}=\lim _{j} x_{i}^{j}$. The sequence $\left\{\widetilde{x}_{i}\right\}$ is very regular as all the $j$ bands in $\left\{x_{i}^{j}\right\}$ were very regular. It dominates $\left\{x_{i}\right\}$ since each of the $\left\{x_{i}^{j}\right\}$ dominates $\left\{x_{i}\right\}$. Thus the lemma is true.

\section{Phase transition on the randomly stretched lattice}

We show that if $\left\{x_{i}\right\}$ and $\left\{y_{i}\right\}$ are very regular then the stretched lattice determined by $\left\{x_{i}\right\}$ and $\left\{y_{i}\right\}$ has an infinite cluster for $p$ close to 1 a.s. We will do this by mimicking the proof that percolation on the regularly stretched lattice has an infinite cluster for $p$ close to 1 a.s. This is enough to establish a phase transition as Jonasson, Mossel, and Peres have proven that if $p$ is sufficiently close to one then there is no infinite cluster a.s.

Fix $\left\{x_{i}\right\}$ and $\left\{y_{i}\right\}$ which are very regular. Let $\omega \in\{0,1\}^{E\left(\mathbb{Z}^{2}\right)}$ be the realization of percolation. We will inductively define what it means for an $n$ box to be good for $\omega$. A cluster $C$ in a subgraph $S$ is a maximal connected subgraph of $S$ such that $\omega(c)=1$ for all $c \in C$. For an $n$ box a crossing cluster is a cluster in the $n$ box which contains vertices on all four edges of the $n$ box. Inductively it will be clear that every good $n$ box contains a crossing cluster. A crossing of a horizontal $(m, n)$ strip $[a, b] \times[c, d]$ is a cluster in the $(m, n)$ strip which contains at least one vertex in $[a, b] \times[c]$ and at least one vertex in $[a, b] \times[d]$.

An $n \leq 200$ box is good if all the edges in the $n$ box are included in $\omega$. Given an $n$ box, $n>200$, suppose we have defined what it means for an $n-1$ box to be good and it being good implies that the $n-1$ box contains a crossing cluster. Let

$$
\begin{aligned}
a_{1}= & \text { the number of } n-1 \text { boxes in the } n \text { box which are not good and } \\
a_{2}= & \text { the number of pairs of neighboring good } n-1 \text { boxes such that the }(n-1, n-1) \\
& \text { strip between them doesn't have a crossing that } \\
& \text { intersects the crossing clusters of the good } n-1 \text { boxes. }
\end{aligned}
$$

Then the $n$ box is good if $a_{1}+a_{2}$ is at most one.

We define a $(9, m)$ tree in a horizontal $(m, n)$ strip inductively in $m$. A $(9, m)$ tree is a set of vertices in the boundary of the $(m, n)$ strip. For any $n$ and any $(2, n) \operatorname{strip}[a, b] \times[c, d]$ and any set $I$ of 9 elements in $[a, b]$ we define two $(9,2)$ trees $T$ and $T^{\prime}$ in a $(2, n)$ strip by

$$
T=\{(i, j): i \in I, j=c\} \text { and } T^{\prime}=\{(i, j): i \in I, j=d .\}
$$


Each $(m, n)$ strip contains at least $(9, m)$ disjoint $(m-1, n)$ strips. A 9 tree in an $(m, n)$ strip is the union of nine $(9, m-1)$ trees in disjoint $(m-1, n)$ strips within the $(m, n)$ strip. Thus each $(9, m)$ tree in an $(m, n)$ strip consists of $9^{m-1}$ vertices. For any $m^{\prime}<m$ the $9^{m-1}$ vertices are in $9^{m-m^{\prime}}\left(9, m^{\prime}\right)$ trees in disjoint $\left(m^{\prime}, n\right)$ strips.

Lemma 4.1 Every pair of good $n$ boxes separated by an $(n, n)$ strip defines at least one $(9, n)$ tree on each side of the $(n, n)$ strip.

Proof. The proof is by induction. Every pair of good 2 boxes separated by an $(2,2)$ strip has at least 9 pairs of vertices, one in each of the 2 boxes, such that every pair is separated by one edge. This forms a $(9,2)$ tree in the $(2,2)$ strip. Every pair of good $n$ boxes separated by an $(n, n)$ strip has at least 11 pairs of $n-1$ boxes, one in each of the $n$ boxes, such that every pair is separated by an $(n-1, n-1)$ strip. In each of the good $n$ boxes at least 10 of the $11 n-1$ boxes are good. Thus every pair of good $n$ boxes separated by an $(n, n)$ strip has at least 9 pairs of $n-1$ boxes, one in each of the boxes, such that every pair is separated by an $(n-1, n-1)$ strip and both the $n-1$ boxes are good. With the induction hypothesis this forms a $(9, n)$ tree in the $(n, n)$ strip.

Many of our calculations will require the following lemma.

Lemma 4.2 For any $c, p_{1}, \ldots, p_{n}, 0<p_{i}<1$, and $a=\sum_{1}^{n} p_{i}$

$$
1-\prod_{1}^{n}\left(1-p_{i}\right) \geq \min \left(1-e^{-c}, \frac{a}{c}\left(1-e^{-c}\right)\right) .
$$

Proof. We have that $\sum_{1}^{n}-p_{i}=-a$ and $\sum_{1}^{n} \ln \left(1-p_{i}\right) \leq a$. Thus

$$
\prod_{1}^{n}\left(1-p_{i}\right) \leq e^{-a}
$$

and

$$
1-\prod_{1}^{n}\left(1-p_{i}\right) \geq 1-e^{-a}
$$

The inequality

$$
1-z^{d} \geq d(1-z)
$$

holds for $0 \leq z, d \leq 1$. If $a \leq c$ then applying this with $z=e^{-c}$ and $d=a / c$ gives

$$
1-\prod_{1}^{n}\left(1-p_{i}\right) \geq 1-e^{-a}=1-\left(e^{-c}\right)^{a / c} \geq \frac{a}{c}\left(1-e^{-c}\right) .
$$


For any set $T \in \mathbb{Z}^{2}$ define

$$
R(T)=\left\{(x, y) \in \mathbb{Z}^{2}: \text { there exists } x^{\prime} \in \mathbb{Z} \text { such that }\left(x^{\prime}, y\right) \in T\right\} .
$$

For any set $V \in \mathbb{Z}$ define

$$
R(V)=\left\{(x, y) \in \mathbb{Z}^{2}: y \in V\right\} .
$$

We can define $C(T)$ and $C(V)$ in an analogous manner.

For the rest of the paper we fix the following. Let $B$ be any $n$ box. Let $B_{1}=\left[a_{1}, b_{1}\right] \times$ $\left[c_{1}, d_{1}\right]$ and $B_{2}=\left[a_{1}, b_{1}\right] \times\left[c_{2}, d_{2}\right]$ be good $n$ boxes. Let $\bar{S}=\left[a_{1}, b_{1}\right] \times\left[d_{1}, c_{2}\right]$ be the $(n, n)$ strip between $B_{1}$ and $B_{2}$. Let $R_{1} \subset R\left(d_{1}\right)$ and $R_{2} \subset R\left(c_{2}\right)$ be two $(9, n)$ trees defined by the crossing clusters of $B_{1}$ and $B_{2}$. Let $S=[\tilde{a}, \tilde{b}] \times[\tilde{c}, \tilde{d}]$ be a $(\lfloor 2 n / 3\rfloor, n)$ strip, $T \subset R(\tilde{d})$ be a $(9,\lfloor 2 n / 3\rfloor)$ tree in $S$ and $T^{\prime} \subset C(T) \cap R(\tilde{c})$ be a collection of $(9, k)$ trees in $S$ with $k \leq\lfloor 2 n / 3\rfloor$.

Lemma 4.3 There exists $p_{c}<1$ such that if $p>p_{c}$ then

1. $P_{p}(B$ is good $)>1-4^{-n}$ and

2. $P_{p}\left(\exists\right.$ a crossing of $S$ that intersects $T$ and $\left.T^{\prime}\right)>\frac{\left|T^{\prime}\right|}{9^{\lfloor 2 n / 3\rfloor}}$.

3. $P\left(\exists\right.$ a crossing of $\bar{S}$ intersecting $R_{1}$ and $\left.R_{2}\right) \geq 1-4^{-n}$.

The proof is by induction with base case $n=200$. Statement 1 is similar to the induction hypothesis for the regularly stretched lattice. Statement 3 is also similar to a step for the regularly stretched lattice. It does not work as an inductive step. Statement 2 is introduced because it is possible to induct on this statement. Statement 3 then follows easily from statement 2. When the height of $S$ is one $(\tilde{c}=\tilde{d})$ the proof of 2 is a simple calculation. The proof of statement 2 when the height of $S$ is greater than one is the most complicated part of the proof of Lemma 4.3. Before we prove Lemma 4.3 we require a few additional lemmas.

Now we consider the case that the height of $S$ is greater than one. Because $\left\{x_{i}\right\}$ and $\left\{y_{i}\right\}$ are very regular $S$ has the following structure. We can break $S$ up into 3 parts. On the bottom we have a $(\lfloor 2 n / 3\rfloor, m)$ strip $S_{1}=[\tilde{a}, \tilde{b}] \times\left[\tilde{c}, \tilde{c}^{\prime}\right]$. On the top we have a $(\lfloor 2 n / 3\rfloor, r)$ band $S_{2}=[\tilde{a}, \tilde{b}] \times\left[\tilde{d^{\prime}}, \tilde{d}\right]$. In the middle are up to 384 rows of $q$ boxes separated by $l$ bands with labels $q$. By the definition of very regular and the way the labels were assigned to bands $m, r>q$ and

$$
m+r-\lfloor q / 3\rfloor-n=0 \text { or } 1 .
$$

In particular if $q>100$ then

$$
\lfloor 2 n / 3\rfloor>\lfloor 2 m / 3\rfloor+\lfloor 2 r / 3\rfloor-q+30 .
$$

The outline of our argument is as follows. If

1. there are "enough" crossings of $S_{1}$ which intersect $T^{\prime}$, 
2. there is at least one crossing of $S_{2}$ which intersects $T$,

3. there exists $v$ contained in a crossing of $S_{1}$ which intersects $T^{\prime}$ and $w$ contained in a crossing of $S_{2}$ which intersects $T$ such that $v$ and $w$ are contained in a column of $q$ boxes, and

4. $v$ and $w$ are connected

then there exists a crossing of $S$ intersecting $T^{\prime}$ and $T$.

In Lemma 4.4 we bound from below the probability that there is at least one crossing of $S_{1}$ intersecting $T^{\prime}$. We define what "enough" is in line 7. In Lemma 4.5 we give a lower bound on the probability of 1 . Then we use Lemma 4.4 to bound the probability of 2 and 3 conditioned on 1 occurring. Then we bound the probability of 4 in Lemma 4.6. Then the proof of Lemma 4.3 part 2 is done by combining the above bounds.

Let $S^{\prime}=\left[a^{\prime}, b^{\prime}\right] \times\left[c^{\prime}, d^{\prime}\right]$ be a $(J, j)$ strip with $j \leq n$ and $J \geq\lfloor 2 j / 3\rfloor$. Let $\hat{S}=\cup \hat{S}_{i}$ be a union of $(l, j)$ strips in $S^{\prime}$. We write $\hat{S}_{i}=\left[f_{i}, g_{i}\right] \times\left[c^{\prime}, d^{\prime}\right]$. Let $\bar{T} \subset R\left(d^{\prime}\right)$ be a $(9, J)$ tree in $\bar{S}$ which intersects each $\hat{S}_{i}$ in a $(9,\lfloor 2 j / 3\rfloor)$ tree. Let $\hat{T} \subset C(\bar{T}) \cap R\left(c^{\prime}\right)$ be a union of $(9, l)$ trees in disjoint $(l, j)$ strips in $\hat{S}$ where $l \leq\lfloor 2 j / 3\rfloor$.

Lemma 4.4 Suppose the conclusions of Lemma 4.3 are satisfied for $j \leq n-1$. Then

$$
P(\exists \text { a crossing of } \hat{S} \text { intersecting } \hat{T} \text { and } \bar{T}) \geq \min \left(.9, \frac{|\hat{T}|}{3 \cdot 9^{\lfloor 2 j / 3\rfloor}}\right) \text {. }
$$

Proof. $\hat{T}$ is the union of $(9, l)$ trees so $\hat{T}=\bigcup T_{i}$ where each $T_{i}$ is a union of $(9, l)$ trees in $(\lfloor 2 j / 3\rfloor, j)$ strip $S_{i}$. By the induction hypothesis we have

$$
P\left(\exists \text { a crossing of } \hat{S}_{i} \text { intersecting } \hat{T}_{i} \text { and } \bar{T}\right) \geq \frac{\left|\hat{T}_{i}\right|}{9\lfloor 2 j / 3\rfloor} .
$$

By Lemma 4.2 with $c=3$

$$
\begin{aligned}
P(\exists \text { a crossing of } \hat{S} \text { intersecting } \hat{T} \text { and } \bar{T}) & \geq \min \left(1-e^{-3}, \frac{\Sigma\left|\hat{T}_{i}\right|}{3 \cdot 9^{\lfloor 2 j / 3\rfloor}}\right) \\
& \geq \min \left(.9, \frac{|\hat{T}|}{3 \cdot 9\lfloor 2 j / 3\rfloor}\right) .
\end{aligned}
$$

We have defined a $(\lfloor 2 n / 3\rfloor, n)$ strip $S$, a $(9,\lfloor 2 n / 3\rfloor)$ tree $T^{\prime}$, a union of $(9, k)$ trees $T^{\prime}$, and constants $q, m$ and $k$. Let $q^{*}=\max (100, q)$ and $M=\max (\lfloor 2 m / 3\rfloor, 100, q)$. Also let $k^{\prime}=\min (k,\lfloor 2 m / 3\rfloor)$. Given $T$ define $\bar{T}=R\left(\tilde{c}^{\prime}\right) \cap C(T)$. Define $\widetilde{T}$ to be the union of the 
$\left(9, q^{*}\right)$ trees in $\bar{T}$ such that for each $\widetilde{T}_{i} \subset \widetilde{T}$ there exists $\tilde{v}_{i} \in \widetilde{T}_{i}, v_{i} \in T^{\prime} \cap C\left(\widetilde{T}_{i}\right)$ and a crossing of $S_{1}$ containing $\tilde{v}_{i}$ and $v_{i}$. Define $*$ to be the event that

$$
|\widetilde{T}| \geq \max \left(\frac{\left|T^{\prime}\right| 9^{q^{*}-1}}{1000 \cdot 9^{M}}, 9^{q^{*}-1}\right)
$$

Lemma 4.5 If Lemma 4.3 is satisfied for $j \leq n-1$ then

$$
P(*)>\min \left(.9, \frac{\left|T^{\prime}\right|}{100 \cdot 9^{\lfloor 2 m / 3\rfloor}}\right) .
$$

Proof. We break the proof up into cases based on the size of $\left|T^{\prime}\right|$ and whether $M=q^{*}$, $M=100$, or $M=\lfloor 2 m / 3\rfloor$. If $\left|T^{\prime}\right| \leq 1000 \cdot 9^{\lfloor 2 m / 3\rfloor}$ and $M=\lfloor 2 m / 3\rfloor$ then by Lemma 4.4

$$
P(*) \geq \min \left(.9, \frac{\left|T^{\prime}\right|}{3 \cdot 9^{\lfloor 2 m / 3\rfloor}}\right) .
$$

If $\left|T^{\prime}\right| \leq 1000 \cdot 9^{M}$ and $M=q^{*}$ then we write $T^{\prime}=\bigcup_{i=1}^{N} T_{i}$ where each $T_{i}$ is a union of $\left(9, k^{\prime}\right)$ trees in a $(\lfloor 2 m / 3\rfloor, m)$ strip. Then for all $i$ by Lemma 4.4

$$
P\left(\exists \text { a crossing intersecting } T_{i} \text { and } \bar{T}\right) \geq \min \left(.9, \frac{\left|T_{i}\right|}{3 \cdot 9^{\lfloor 2 m / 3\rfloor}}\right) .
$$

If for one $i$ the minimum is .9 then we are done. Otherwise by Lemma 4.2 we have

$$
P\left(\exists \text { a crossing intersecting } T^{\prime} \text { and } \bar{T}\right)>\min \left(.9, \frac{\left|T^{\prime}\right|}{9 \cdot 9^{\lfloor 2 m / 3\rfloor}}\right) .
$$

If $\left|T^{\prime}\right|>1000 \cdot 9^{M}$ then write $T^{\prime}=\bigcup_{i=1}^{N} T_{i}^{\prime}$ where each $T_{i}^{\prime}$ is a union of $\left(9, k^{\prime}\right)$ trees in a union of $(M, m)$ strips. Do this in such a way that for each $i$

$$
3 \cdot 9^{M} \leq\left|T_{i}^{\prime}\right|=4 \cdot 9^{M} .
$$

and if $i \neq j$ the unions of $(M, m)$ strips for $i$ and $j$ are disjoint. Thus $N$ is at least

$$
\frac{\left|T^{\prime}\right|}{4 \cdot 9^{M}} \geq 100
$$

By the fact that $\left|T_{i}^{\prime}\right| \geq 3 \cdot 9^{M}$ we have that

$$
P\left(\exists \text { a crossing of } \widetilde{S}_{i} \text { intersecting } T_{i}^{\prime} \text { and } T^{*}\right) \geq .9 .
$$

Thus we have $N \geq 100$ independent events with probability greater than or equal to .9. The probability that at least $\lceil N / 10\rceil$ of these events happen is greater than the probability that 11 events happen with $N=100$. This probability is at least .9. 
Finally if $M=100$ then $q \leq 100$ and $m \leq 150$. Thus

$$
P(*) \geq 1-4^{-200} \text {. }
$$

Let $G$ be a graph which is composed of the union of a column of $q$ boxes, $[e, f] \times\left[g_{1}, h_{1}\right]$ through $[e, f] \times\left[g_{l}, h_{l}\right]$, where $q \geq 200$ and $l \leq 384$. Also in $G$ are horizontal $(q, q)$ strips $[e, f] \times\left[h_{1}, g_{2}\right]$ through $[e, f] \times\left[h_{l-1}, l_{k}\right]$. Thus $G=[e, f] \times\left[g_{1}, h_{l}\right]$. We call such a graph a column of $q$ boxes. Let $v \subset R\left(g_{1}\right)$ and $w \subset R\left(h_{l}\right)$ be vertices on the bottom and top of $G$. We say $G$ is normal for $v$ and $w$ if there exists a cluster in $G$ connecting $v$ and $w$.

Lemma 4.6 Suppose the conclusions of Lemma 4.3 are satisfied for $q \leq n$. Then

$$
P(G \text { is normal for } v \text { and } w) \geq .99
$$

Proof. $G$ is normal for $v$ and $w$ if

1. all of the $q$ boxes in $G$ are good,

2. $v$ and $w$ are in the crossing clusters of their respective $q$ boxes, and

3. all of the $(q, q)$ strips in $G$ have a cluster which connects the crossing clusters of the good $q$ boxes on the top or bottom of the $(q, q)$ strip.

Thus by the induction hypothesis

$$
P(\text { condition } 1 \text { is satisfied })>\left(1-4^{-q}\right)^{384}>1-2^{-100} .
$$

If the $j$ box containing $v$ (or $w$ ) is good for all $j, 200 \leq j \leq q$, then $v$ (or $w$ ) is in the crossing cluster of the $q$ box. Thus

$$
P(\text { condition } 2 \text { is satisfied })>\left(1-2 \sum_{j \geq 200} 4^{-j}\right)>1-4^{-199} .
$$

By Lemma 4.3

$$
P(\text { condition } 3 \text { is satisfied })>\left(1-4^{-q}\right)^{384}>1-2^{-100} \text {. }
$$

Thus

$$
P(G \text { is normal for } v \text { and } w)>1-3 \cdot 2^{-100}>.99 \text {. }
$$

Proof of Lemma 4.3: The proof is by induction. Choose $p_{c}$ such that for $p>p_{c}$ the lemma is true for all $n \leq 200$. Assume that the lemma is true for all $j<n$.

Since $\left\{x_{i}\right\}$ and $\left\{y_{i}\right\}$ are regular there are at most $384^{2} n-1$ boxes in an $n$ box and we have that

$$
P_{p}\left(a_{1}=1\right) \leq 384^{2}\left(4^{-n+1}\right) \leq 4^{10} 4^{-n}
$$


and

$$
P_{p}\left(a_{1} \geq 2\right) \leq\left(384^{2}\right)^{2}\left(4^{-n+1}\right)^{2} \leq 4^{30} 4^{-2 n} \leq 4^{-50} 4^{-n} .
$$

There are at most $2(384)^{2}(n-1, n-1)$ strips in an $n$ box. If such a strip is between two good $n-1$ boxes then the probability that there exists a crossing which connects to both the crossing clusters of the good $n$ boxes is calculated as follows.

By the induction hypothesis

$$
P_{p}\left(a_{2} \geq 2\right) \leq\left(2(384)^{2}\right)^{2}\left(4^{-n+1}\right)^{2} \leq 4^{20} 4^{-2 n} \leq 4^{-50} 4^{-n}
$$

and

$$
P_{p}\left(a_{2} \geq 1 \mid a_{1}=1\right) \leq 2(384)^{2}\left(4^{-n+1}\right) \leq 4^{20} 4^{-n}
$$

Thus

$$
\begin{aligned}
P_{p}\left(a_{1}+a_{2} \geq 2\right) & \leq P_{p}\left(a_{1} \geq 2\right)+P_{p}\left(a_{2}=1 \mid a_{1}=1\right) \cdot P_{p}\left(a_{1}=1\right)+P_{p}\left(a_{2} \geq 2\right) \\
& \leq 2\left(4^{-50}\right) 4^{-n}+4^{30} 4^{-2 n} \\
& \leq 4^{-n}
\end{aligned}
$$

This completes the proof of the first statement.

Now we consider the second statement. First we must consider the case that the height of $S$ is one. In this case the probability is easy to calculate. There are $\left|T^{\prime}\right|$ edges which would form an appropriate crossing if they were open. Thus

$$
\begin{aligned}
P_{p}\left(\exists \text { a cluster in } S \text { which intersects } T \text { and } T^{\prime}\right) & \geq 1-\left(1-p^{n}\right)^{\left|T^{\prime}\right|} \\
& \geq \min \left(1-e^{-1},\left|T^{\prime}\right| p^{n}\left(1-e^{-1}\right)\right) \\
& \geq \frac{\left|T^{\prime}\right|}{9^{\lfloor 2 n / 3\rfloor}}
\end{aligned}
$$

by Lemma 4.2. Next it is easy to see that if either $m<200$ or $r<200$ then $q<200$ and that the induction hypothesis is easily proven.

Now we consider the case when $m, r \geq 200$ and the height of $S$ is greater than one. If $q \geq 100$ and

1. the event $*$ happens on $S_{1}$. This forms $\widetilde{T} \subset C(T) \cap R\left(\tilde{c}^{\prime}\right)$.

2. Set $T^{*}=C(\widetilde{T}) \cap R\left(\tilde{d}^{\prime}\right)$. There exists a crossing of $S_{2}$ intersecting $T^{*}$ and $T$. This gives us $v \in \widetilde{T}$ and $w \in T^{*}$. They are separated by a column of $q$ boxes. And

3. the column of $q$ boxes separating $v$ and $w$ is normal for $v$ and $w$

then there exists a crossing of $S$ that intersects $T$ and $T^{\prime}$.

By Lemma 4.5

$$
P(1)=P(*) \geq \min \left(.9, \frac{\left|T^{\prime}\right|}{100 \cdot 9^{\lfloor 2 m / 3\rfloor}}\right) .
$$


If $*$ occurs then we get

$$
|\tilde{T}| \geq \max \left(9^{q^{*}-1}, \frac{9^{q^{*}-1}\left|T^{\prime}\right|}{1000 \cdot 9^{M}}\right) .
$$

If $\left|T^{\prime}\right| \leq 1000 \cdot 9^{M}$ then $\left|T^{*}\right|=|\tilde{T}| \geq 9^{q^{*-1}}$ and by Lemmas 4.5 and 4.6

$$
\begin{aligned}
P(2 \text { and } 3 \mid 1) & \geq P\left(\exists \text { a crossing of } S_{2} \text { intersecting } T^{*} \text { and } T|| T^{*} \mid=9^{q^{*}-1}\right)(.99) \\
& \geq(.99) \min \left(.9, \frac{9^{q^{*}-1}}{3 \cdot 9^{\lfloor 2 r / 3\rfloor}}\right) \\
& \geq \min \left(.8, \frac{9^{q^{*}-1}}{4 \cdot 9^{\lfloor 2 r / 3\rfloor}}\right) .
\end{aligned}
$$

If $\left|T^{\prime}\right|<100 \cdot 9^{\lfloor 2 m / 3\rfloor}$ then

$P\left(\exists\right.$ a crossing of $S$ intersecting $T^{\prime}$ and $\left.T\right) \geq P(1) \cdot P(2$ and $3 \mid 1)$

$$
\begin{aligned}
& \geq \frac{\left|T^{\prime}\right|}{100 \cdot 9^{\lfloor 2 m / 3\rfloor}} \min \left(.8, \frac{9^{q^{*}-1}}{4 \cdot 9^{\lfloor 2 r / 3\rfloor}}\right) \\
& \geq \min \left(\frac{\left|T^{\prime}\right|}{200 \cdot 9\lfloor 2 m / 3\rfloor}, \frac{9^{q^{*}-1}\left|T^{\prime}\right|}{400 \cdot 9^{\lfloor 2 m / 3\rfloor} 9^{\lfloor 2 r / 3\rfloor}}\right) \\
& \geq \frac{\left|T^{\prime}\right|}{9^{\lfloor 2 n / 3\rfloor}} .
\end{aligned}
$$

If $100 \cdot 9^{\lfloor 2 m / 3\rfloor} \leq\left|T^{\prime}\right| \leq 1000 \cdot 9^{M}$ then

$$
\begin{aligned}
P\left(\exists \text { a crossing of } S \text { intersecting } T^{\prime} \text { and } T\right) & \geq P(1) \cdot P(2 \text { and } 3 \mid 1) \\
& \geq(.9) \min \left(.8, \frac{9^{q^{*}-1}}{4 \cdot 9^{\lfloor 2 r / 3\rfloor}}\right) \\
& \geq \min \left(.5, \frac{9^{q^{*} / 3} 1000 \cdot 9^{\lfloor 2 m / 3\rfloor}}{9^{\lfloor 2 r / 3\rfloor+\lfloor 2 m / 3\rfloor-q^{*} / 2}}\right) \\
& \geq \min \left(.5, \frac{1000 \cdot 9^{M}}{9\lfloor 2 n / 3\rfloor}\right) \\
& \geq \min \left(.5, \frac{\left|T^{\prime}\right|}{9^{\lfloor 2 n / 3\rfloor}}\right) \\
& \geq \frac{\left|T^{\prime}\right|}{9^{\lfloor 2 n / 3\rfloor}} .
\end{aligned}
$$

If $\left|T^{\prime}\right| \geq 1000 \cdot 9^{M}$ and * occurs then

$$
|\widetilde{T}|=\left|T^{*}\right| \geq \frac{\left|T^{\prime}\right| 9^{q^{*}-1}}{1000 \cdot 9^{M}}
$$


and by Lemma 4.4

$$
\begin{aligned}
P(2 \mid 1) & \geq P\left(\exists \text { a crossing of } S_{2} \text { intersecting } T^{*} \text { and } T|| T^{*} \mid=\frac{\left|T^{\prime}\right| 9^{q^{*}-1}}{1000 \cdot 9^{M}}\right) \\
& \geq \min \left(.9, \frac{\left|T^{\prime}\right|}{3000 \cdot 9^{M+\lfloor 2 r / 3\rfloor-q^{*}+1}}\right) \\
& \geq \min \left(.9, \frac{2\left|T^{\prime}\right|}{9^{\lfloor 2 n / 3\rfloor}}\right) \\
& \geq \frac{2\left|T^{\prime}\right|}{9^{\lfloor 2 n / 3\rfloor}} .
\end{aligned}
$$

By Lemma 4.6

$$
P(3 \mid 1 \text { and } 2) \geq .99
$$

If $\left|T^{\prime}\right| \geq 1000 \cdot 9^{M}$ then

$$
\begin{aligned}
P\left(\exists \text { a crossing of } S \text { intersecting } T^{\prime} \text { and } T\right) & \geq P(1) \cdot P(2 \mid 1) \cdot P(3 \mid 1 \text { and } 2) \\
& \geq(.9) \frac{2\left|T^{\prime}\right|}{9^{\lfloor 2 n / 3\rfloor}}(.99) \\
& \geq \frac{\left|T^{\prime}\right|}{9^{\lfloor 2 n / 3\rfloor}} .
\end{aligned}
$$

For any two points $\left(x_{1}, y_{1}\right),\left(x_{2}, y_{2}\right) \in \mathbb{Z}^{2}$ we say the rectangle formed by $v$ and $w$ is the graph of all edges where both of vertices have $x$ coordinate between $x_{1}$ and $x_{2}$ and have $y$ coordinate between $y_{1}$ and $y_{2}$. If $q<100$ we substitute 3 with

$\left.3^{\prime}\right)$ all edges in the rectangle formed by $v$ and $w$ are open.

Then everything goes through as above. This is because the rectangle between $v$ and $w$ is a portion of a 200 box. This completes the proof of statement 2 .

Finally we prove statement 3 . The $(9, n)$ trees $R$ and $R^{\prime}$ defines a set of $9^{n-\lfloor 2 n / 3\rfloor}$ $(\lfloor 2 n / 3\rfloor, n)$ strips in $\bar{S}$. Let $\tilde{S}$ be one. By the induction hypothesis the probability that there is a crossing of $\tilde{S}$ which intersects $R$ and $R^{\prime}$ is at least $\frac{1}{9}$. As there are $9^{n-\lfloor 2 n / 3\rfloor}$ of these $(\lfloor 2 n / 3\rfloor, n)$ strips and the events that there does not exist crossings that intersect both $R$ and $R^{\prime}$ are independent. The probability that there exists an appropriate crossing is at least

$$
\begin{aligned}
1-\left(\frac{8}{9}\right)^{9^{n-\lfloor 2 n / 3\rfloor}} & \geq \min \left(1-e^{-2 n}, \frac{9^{n-\lfloor 2 n / 3\rfloor-1}}{2 n}\left(1-e^{-2 n}\right)\right) \\
& \geq 1-e^{-2 n} \\
& \geq 1-4^{-n} .
\end{aligned}
$$

Theorem 4.1 There exists $p_{c}<1$ such that for almost every $\left\{x_{i}\right\}$ and $\left\{y_{i}\right\}$ and all $p>p_{c}$

$$
P_{p}(\exists \text { an infinite cluster })=1 \text {. }
$$


Proof. By Lemma 3.8 there exists $\left\{\bar{x}_{i}\right\}$ and $\left\{\bar{y}_{i}\right\}$ which are very regular and $\bar{x}_{i} \geq x_{i}$ and $\bar{y}_{i} \geq y_{i}$ for all $i$. Thus the estimates in Lemma 4.3 apply. Let $p_{c}$ be as in Lemma 4.3. It is easy to check that if $v$ and $w$ are two vertices in the same $n$ box, $v$ is in a good $j$ box for all $j \leq n$ and $w$ is in a good $j$ box for all $j \leq n$, then $v$ and $w$ are the same cluster. Thus if $v$ is in a good $n$ box for all $n$ then $v$ is in an infinite cluster. Thus

$$
\begin{aligned}
P_{p}(\exists \text { an infinite cluster }) & \geq P_{p}(0 \text { is in a good } n \text { box for all } n) \\
& \geq 1-\sum_{n=200}^{\infty} 4^{-n} \\
& \geq 1-4^{-199} .
\end{aligned}
$$

As $P_{p}(\exists$ an infinite cluster $)$ equals 0 or 1

$$
P_{p}(\exists \text { an infinite cluster })=1 .
$$

Theorem 4.2 For percolation on the randomly stretched lattice in $\mathbb{Z}^{2}$ there exists a value $p_{c}, 0<p_{c}<1$ such that if $p<p_{c}$ then

$$
P_{p}(\exists \text { an infinite open cluster })=0 .
$$

If $p>p_{c}$ then

$$
P_{p}(\exists \text { an infinite open cluster })=1 \text {. }
$$

Proof. It follows from [6] that if $p$ is sufficiently close to 0 then there is no infinite cluster a.s. Thus Theorem 4.1 completes the proof.

Acknowledgment: I would like to thank David Levin and Yuval Peres for introducing me to the problem. I would also like to thank Yuval Peres and Eric Babson for helpful conversations.

\section{References}

[1] P. Balister, B. Bollobs and A. Stacey. Dependent percolation in two dimensions. Probab. Theory Related Fields 117 (2000), no. 4, 495-513.

[2] J. Chayes, L. Chayes and R. Durrett. Connectivity properties of Mandelbrot's percolation process. Probab. Theory Related Fields 77 (1988), no. 3, 307-324.

[3] I. Ferreira. The probability of survival for the biased voter model in a random environment. Stochastic Process. Appl. 34 (1990), no. 1, 25-38.

[4] P. Gacs. Clairvoyant Scheduling of Random Walks preprint. 
[5] P. Gacs. Compatible Sequences and a Slow Winkler Percolation preprint.

[6] J. Jonasson, E. Mossel and Y. Peres. Percolation in a dependent random environment. Random Struct. Alg. 16 (2000), no. 4, 333-343.

[7] A. Klein. Extinction of contact and percolation processes in a random environment. Ann. Probab. 22 (1994), no. 3, 1227-1251.

[8] T. Liggett. The survival of one-dimensional contact processes in random environments. Ann. Probab. 20 (1992), no. 2, 696-723.

[9] B. McCoy and T. Wu. Theory of a two-dimensional Ising model with random impurities. I. Thermodynamics. Phys. Rev. (2) 1761968 631-643.

[10] B. McCoy and T. Wu. Theory of a two-dimensional Ising model with random impurities. II. Spin correlation functions. Phys. Rev. (2) 1881969 982-1013.

[11] C. Newman and S. Volchan. Persistent survival of one-dimensional contact processes in random environments. Ann. Probab. 24 (1996), no. 1, 411-421.

[12] P. Winkler Dependent percolation and colliding random walks. Random Structures Algorithms 16 (2000), no. 1, 58-84. 